\title{
Local density of Caputo-stationary functions of any order*
}

\author{
Alessandro Carbotti ${ }^{\dagger}$ Serena Dipierro ${ }^{\ddagger}$ and Enrico Valdinoci ${ }^{\S}$
}

November 2, 2018

\begin{abstract}
We show that any given function can be approximated with arbitrary precision by solutions of linear, time-fractional equations of any prescribed order.

This extends a recent result by Claudia Bucur, which was obtained for time-fractional derivatives of order less than one, to the case of any fractional order of differentiation.

In addition, our result applies also to the $\psi$-Caputo-stationary case, and it will provide one of the building blocks of a forthcoming paper in which we will establish general approximation results by operators of any order involving anisotropic superpositions of classical, space-fractional and time-fractional diffusions.
\end{abstract}

\section{Introduction}

The goal of this paper is to show that every function can be approximated arbitrarily well by functions that satisfy a homogeneous equation driven by the Caputo derivative of any positive order.

When the order of the Caputo derivative is less than one, this type of statement has been recently proved in [Buc17]. In this sense, our result here is the extension of the main theorem in [Buc17] to higher order Caputo fractional derivatives.

More precisely, we denote by $\mathbb{N}$ the set of natural numbers (starting from 1 ) and $\mathbb{N}_{0}:=$ $\mathbb{N} \cup\{0\}$ (in this way, $\mathbb{N}=\mathbb{N}_{0} \backslash\{0\}$ ). Following [Cap08], given

$$
a \in \mathbb{R} \cup\{-\infty\}, k \in \mathbb{N} \text { and } \alpha \in(k-1, k),
$$

one defines the Caputo derivative of initial point $a$ and order $\alpha$ by

$$
D_{a}^{\alpha} u(t):=\frac{1}{\Gamma(k-\alpha)} \int_{a}^{t} \frac{u^{(k)}(\tau)}{(t-\tau)^{\alpha-k+1}} d \tau
$$

*Supported by the Australian Research Council Discovery Project 170104880 NEW "Nonlocal Equations at Work". The authors are members of INdAM/GNAMPA.

${ }^{\dagger}$ Dipartimento di Matematica e Fisica, Università del Salento, Via Per Arnesano, 73100 Lecce, Italy. alessandro.carbotti@unisalento.it

${ }_{\ddagger}^{\ddagger}$ Department of Mathematics and Statistics, University of Western Australia, 35 Stirling Highway, Crawley WA 6009, Australia. serena.dipierro@uwa.edu.au

$\S$ Department of Mathematics and Statistics, University of Western Australia, 35 Stirling Highway, Crawley WA 6009, Australia, and Istituto di Matematica Applicata e Tecnologie Informatiche, Consiglio Nazionale delle Ricerche, Via Ferrata 1, 27100 Pavia, Italy, and Dipartimento di Matematica, Università degli studi di Milano, Via Saldini 50, 20133 Milan, Italy. enrico@mat.uniroma3.it 
where $\Gamma$ is the Euler's Gamma-Function.

In addition, if $I \subseteq \mathbb{R}$ is an interval, we define the space

$$
A C^{k-1}(I):=\left\{f \in C^{k-1}(I) \text { s.t. } f, f^{\prime}, \ldots, f^{(k-1)} \in A C(I)\right\},
$$

where $C^{k-1}(I)$ denotes the space of $(k-1)$-times continuously differentiable functions on $I$, and $A C(I)$ denotes the space of absolutely continuous functions on $I$.

Given $t>a, k \in \mathbb{N}, \beta>0$, and $f:[a,+\infty) \rightarrow \mathbb{R}$, we also define the function

$$
(a, t) \ni \tau \mapsto \Theta_{k, \beta, f, t}(\tau):=f^{(k)}(\tau)(t-\tau)^{k-\beta-1}
$$

and we set

$$
\begin{array}{r}
C_{a}^{k, \beta}:=\left\{f: \overline{(a,+\infty)} \rightarrow \mathbb{R} \text { s.t. } f \in A C^{k-1}(\overline{(a, t)})\right. \\
\text { and } \left.\Theta_{k, \beta, f, t} \in L^{1}((a, t)), \text { for all } t>a\right\} .
\end{array}
$$

We observe that the Caputo derivative in (1.2) is well defined for all $u$ belonging to $C_{a}^{k, \alpha}$. In this setting, we have the following density result:

Theorem 1.1. Let $h \in \mathbb{N}_{0}, k \in \mathbb{N}$, and $\alpha \in(k-1, k)$.

Then, for every $f \in C^{h}([0,1])$ and $\epsilon>0$, there exist $a<0$ and $u \in C_{a}^{k, \alpha}$ such that

$$
\begin{array}{ll} 
& D_{a}^{\alpha} u(t)=0 \quad \text { in } \quad[0,+\infty) \\
\text { and } \quad & \|u-f\|_{C^{h}([0,1])}<\epsilon
\end{array}
$$

Theorem 1.1 lies in the research line of approximation results with solutions of nonlocal equations. The first result in this direction was obtained in [DSV17], where it was established that any given function can be locally approximated with arbitrary precision by functions with vanishing fractional Laplacian. This result has been also extended in [DSV18] to take into account also evolution equations, and in general equations which contain different types of diffusion in different coordinate variables.

When $\alpha \in(0,1)$, Theorem 1.1 has been recently obtained in [Buc17]. Furthermore, Theorem 1.1 will constitute one of the main building blocks for the forthcoming paper [CDV], in which we will establish a very general density result for solutions of operators taking into account both classical and fractional derivatives of any order and of both time-fractional and space-fractional types.

We also remark that Caputo derivatives possess a number of concrete applications in describing processes with memory, see e.g. [Sam02] and the references therein, hence we think that it is quite interesting that the set of solutions of linear Caputo-type equations is shown by Theorem 1.1 to be so abundant to shadow the profile of any prescribed function, also independently on any geometric constraint.

The proof of Theorem 1.1 will rely on an appropriate "derivative spanning" method introduced in [DSV17]. Roughly speaking, the nonlocal effect produced by the operator causes a fractional type boundary behavior which is persistent for all the derivatives of the solutions. Then, this phenomenon in turn implies that the derivative jet at a given point is essentially arbitrary, and the desired result follows by rescaling.

To make such argument work, one needs to construct a suitable solution with a very precise control on every boundary derivatives. In our setting, this goal will be achieved by 
a careful analysis of the linear equation, in terms of explicit representation formulas and asymptotic analysis.

It is also interesting to point out that a simple variant of Theorem 1.1 comprises the case of more general nonlocal operators of time-fractional type. To describe this generalized setting, we consider the functional space

$$
\Psi_{k}:=\left\{\psi \in C^{k}(\mathbb{R}) \text { s.t. } \psi^{\prime}(x)>0 \text { for any } x \in \mathbb{R}\right\} .
$$

Given $\psi \in \Psi_{k}$, following [Alm17, Tar13], one can introduce a time-fractional derivative with respect to $\psi$ and initial point $a \in \mathbb{R} \cup\{-\infty\}$, defined by

$$
D_{a}^{\alpha, \psi} u(t):=\frac{1}{\Gamma(k-\alpha)} \int_{a}^{t} \frac{u_{\psi}^{(k)}(\tau)}{(\psi(t)-\psi(\tau))^{\alpha-k+1}} \psi^{\prime}(\tau) d \tau
$$

where

$$
u_{\psi}^{(k)}(\tau):=\left(\frac{1}{\psi^{\prime}(\tau)} \frac{d}{d \tau}\right)^{k} u(\tau)=\underbrace{\left(\frac{1}{\psi^{\prime}(\tau)} \frac{d}{d \tau}\right) \cdots\left(\frac{1}{\psi^{\prime}(\tau)} \frac{d}{d \tau}\right)}_{k \text { times }} u(\tau) .
$$

The setting in (1.8) comprises, as a particular case, the Caputo fractional derivative given by (1.2) (notice indeed that (1.8) reduces to $(1.2)$ when $\psi(t):=t)$.

In this context, we obtain from Theorem 1.1 that $\psi$-Caputo-stationary functions are locally dense, in the sense made precise by the following result:

Corollary 1.2. Let $h \in \mathbb{N}_{0}, k \in \mathbb{N}$, and $\alpha \in(k-1, k)$. Let also $\psi \in \Psi_{k} \cap C^{h}([0,1])$.

Assume that

$$
\lim _{t \rightarrow-\infty} \psi(t)=-\infty
$$

Then, for any $\epsilon>0$ and any $f \in C^{h}([0,1])$ there exist an initial point $a \in(-\infty, 0)$ and $a$ function $u \in C_{a}^{k, \alpha}$ which satisfies

$$
\begin{array}{ll} 
& D_{a}^{\alpha, \psi} u(t)=0 \quad \text { for any } t \in[0,+\infty) \\
\text { and } \quad & \|u-f\|_{C^{h}([0,1])}<\epsilon .
\end{array}
$$

In the forthcoming Section 2, we will prove Theorem 1.1. From this, we will derive the proof of Corollary 1.2 in Section 3.

\section{Proof of Theorem 1.1}

\subsection{Existence, uniqueness and regularity of solutions of time-fractional equations}

The proof of Theorem 1.1 relies on a series of auxiliary results (here, if not specified, we always implicitly suppose that the setting in (1.1) is assumed). We start with an equivalent formulation of time-fractional equations which highlights the role played by the memory effect: roughly speaking, solving a homogeneous time-fractional equation with some given initial data at $t=a$ is equivalent to solving a non-homogeneous time-fractional equation with initial time $t=b>a$, and the non-homogeneous source in the equation takes into account the memory effect of the period of time $t \in[a, b]$. The precise result that we need is the following: 
Lemma 2.1. Let $b>a$ and $\varphi \in C^{k}([a, b])$. Then, $u \in C_{a}^{k, \alpha}$ satisfies the equation

$$
\left\{\begin{array}{lll}
D_{a}^{\alpha} u(t)=0 & \text { in } & (b,+\infty) \\
u(t)=\varphi(t) & \text { in } & (-\infty, b]
\end{array}\right.
$$

if and only if it satisfies

$$
\left\{\begin{array}{lll}
D_{b}^{\alpha} u(t)=g(t) & \text { in } & (b,+\infty) \\
u(t)=\varphi(t) & \text { in } & (-\infty, b]
\end{array}\right.
$$

where

$$
g(t):=-\frac{1}{\Gamma(k-\alpha)} \int_{a}^{b} \frac{\varphi^{(k)}(\tau)}{(t-\tau)^{\alpha-k+1}} d \tau .
$$

Proof. First of all, we observe that the function $g$ in (2.1) is well defined, since

$$
\Gamma(k-\alpha)|g(t)| \leq \int_{a}^{b} \frac{\left|\varphi^{(k)}(\tau)\right|}{(t-\tau)^{\alpha-k+1}} d \tau \leq \sup _{\tau \in[a, b]}\left|\varphi^{(k)}(\tau)\right| \frac{(t-a)^{k-\alpha}-(t-b)^{k-\alpha}}{k-\alpha},
$$

which is finite for any $t \in[b,+\infty)$.

Using the definition in (1.2), we have that

$$
\begin{aligned}
D_{a}^{\alpha} u(t) & =\frac{1}{\Gamma(k-\alpha)} \int_{b}^{t} u^{(k)}(\tau)(t-\tau)^{k-\alpha-1} d \tau+\frac{1}{\Gamma(k-\alpha)} \int_{a}^{b} u^{(k)}(\tau)(t-\tau)^{k-\alpha-1} d \tau \\
& =\frac{1}{\Gamma(k-\alpha)} \int_{b}^{t} u^{(k)}(\tau)(t-\tau)^{k-\alpha-1} d \tau+\frac{1}{\Gamma(k-\alpha)} \int_{a}^{b} \varphi^{(k)}(\tau)(t-\tau)^{k-\alpha-1} d \tau \\
& =D_{b}^{\alpha} u(t)-g(t) .
\end{aligned}
$$

From this, the desired result plainly follows.

Next lemma gives a representation formula for the solutions of time-fractional equations of any order (when the order is below 1, such a result is related to Volterra-type integral equations, and we provide the details for any order for the facility of the reader). The main representation formula will be given in the forthcoming Lemma 2.3. To this end, we present some ancillary observation on the derivatives of integral identities:

Lemma 2.2. Let $g \in C_{a}^{k, k-\alpha}$, and let, for any $t \geq a$,

$$
v(t):=\int_{a}^{t} g(\tau)(t-\tau)^{\alpha-1} d \tau
$$

Then, $v \in A C^{k-1}([a,+\infty))$, and, for any $t>a$,

$$
v^{(k)}(t)=\int_{a}^{t} g^{(k)}(\tau)(t-\tau)^{\alpha-1} d \tau+\sum_{i=0}^{k-1} \frac{(\alpha+i) \ldots(\alpha+i-k+1) g^{(i)}(a)}{\alpha(\alpha+1) \ldots(\alpha+i)}(t-a)^{\alpha+i-k} .
$$

Proof. Using recursively the Fundamental Theorem of Calculus for absolutely continuous functions (see e.g. Theorem 7.6 in [ADPM11]), we can write

$$
g(t):=\frac{1}{(k-1) !} \int_{a}^{t} g^{(k)}(\sigma)(t-\sigma)^{k-1} d \sigma+\sum_{i=0}^{k-1} \frac{g^{(i)}(a)}{i !}(t-a)^{i} .
$$


As a result, we can write

$$
\begin{gathered}
v(t)=\frac{1}{(k-1) !} \int_{a}^{t}\left[\int_{a}^{\tau} g^{(k)}(\sigma)(\tau-\sigma)^{k-1} d \sigma\right](t-\tau)^{\alpha-1} d \tau \\
+\sum_{i=0}^{k-1} \frac{g^{(i)}(a)}{i !} \int_{a}^{t}(\tau-a)^{i}(t-\tau)^{\alpha-1} d \tau .
\end{gathered}
$$

We also remark that, for every $\sigma \in[a, t)$,

$$
\begin{aligned}
\int_{\sigma}^{t}(\tau & -\sigma)^{k-1}(t-\tau)^{\alpha-1} d \tau=-\frac{1}{\alpha} \int_{\sigma}^{t}(\tau-\sigma)^{k-1} \frac{d}{d \tau}(t-\tau)^{\alpha} d \tau \\
& =\frac{k-1}{\alpha} \int_{\sigma}^{t}(\tau-\sigma)^{k-2}(t-\tau)^{\alpha} d \tau=-\frac{k-1}{\alpha(\alpha+1)} \int_{\sigma}^{t}(\tau-\sigma)^{k-2} \frac{d}{d \tau}(t-\tau)^{\alpha+1} d \tau \\
& =\frac{(k-1)(k-2)}{\alpha(\alpha+1)} \int_{\sigma}^{t}(\tau-\sigma)^{k-3}(t-\tau)^{\alpha+1} d \tau=\ldots \\
& =\frac{(k-1)(k-2) \ldots(k-\ell)}{\alpha(\alpha+1) \ldots(\alpha+\ell-1)} \int_{\sigma}^{t}(\tau-\sigma)^{k-\ell-1}(t-\tau)^{\alpha+\ell-1} d \tau \\
& =\frac{(k-1) !}{\alpha(\alpha+1) \ldots(\alpha+k-2)} \int_{\sigma}^{t}(t-\tau)^{\alpha+k-2} d \tau \\
& =\frac{(k-1) !}{\alpha(\alpha+1) \ldots(\alpha+k-1)}(t-\sigma)^{\alpha+k-1} .
\end{aligned}
$$

In addition, since $\Theta_{k, k-\alpha, g, t} \in L^{1}((a, t))$ for all $t>a$ in view of (1.3) and (1.4), we know that the map

$$
(a, t) \ni \sigma \mapsto g^{(k)}(\sigma)(t-\sigma)^{\alpha+k-1}
$$

belongs to $L^{1}((a, t))$. Hence, we can exploit (2.5) and Fubini's Theorem to see that

$$
\begin{aligned}
& \frac{(k-1) !}{\alpha(\alpha+1) \ldots(\alpha+k-1)} \int_{a}^{t} g^{(k)}(\sigma)(t-\sigma)^{\alpha+k-1} d \sigma \\
= & \int_{a}^{t} g^{(k)}(\sigma)\left[\int_{\sigma}^{t}(\tau-\sigma)^{k-1}(t-\tau)^{\alpha-1} d \tau\right] d \sigma \\
= & \int_{a}^{t}\left[\int_{a}^{\tau} g^{(k)}(\sigma)(\tau-\sigma)^{k-1} d \sigma\right](t-\tau)^{\alpha-1} d \tau .
\end{aligned}
$$

Plugging this information into (2.4), and using also (2.5) once again, we conclude that, for every $t>a$,

$$
\begin{gathered}
v(t)=\frac{1}{\alpha(\alpha+1) \ldots(\alpha+k-1)} \int_{a}^{t} g^{(k)}(\sigma)(t-\sigma)^{\alpha+k-1} d \sigma \\
+\sum_{i=0}^{k-1} \frac{g^{(i)}(a)}{\alpha(\alpha+1) \ldots(\alpha+i)}(t-a)^{\alpha+i} .
\end{gathered}
$$

We can now take derivatives and find that, for each $j \in\{0, \ldots, k-1\}$,

$$
\begin{aligned}
v^{(j)}(t)= & \frac{1}{\alpha(\alpha+1) \ldots(\alpha+k-1-j)} \int_{a}^{t} g^{(k)}(\sigma)(t-\sigma)^{\alpha+k-1-j} d \sigma \\
& +\sum_{i=0}^{k-1} \frac{(\alpha+i) \ldots(\alpha+i-j+1) g^{(i)}(a)}{\alpha(\alpha+1) \ldots(\alpha+i)}(t-a)^{\alpha+i-j} .
\end{aligned}
$$


In particular, when $j:=k-1$,

$v^{(k-1)}(t)=\frac{1}{\alpha} \int_{a}^{t} g^{(k)}(\sigma)(t-\sigma)^{\alpha} d \sigma+\sum_{i=0}^{k-1} \frac{(\alpha+i) \ldots(\alpha+i-k+2) g^{(i)}(a)}{\alpha(\alpha+1) \ldots(\alpha+i)}(t-a)^{\alpha+i-k+1}$.

Taking one more derivative, we obtain (2.2), as desired. Also, from (2.2) and (2.6), we see that $v \in A C^{k-1}([a,+\infty))$.

Now we state a representation result for linear fractional equations of any order.

Lemma 2.3. Let $g \in C_{b}^{k, k-\alpha}$. The problem

$$
\begin{cases}D_{b}^{\alpha} u(t)=g(t) & \text { in } \quad(b,+\infty) \\ u^{(h)}(b)=0 & \text { for any } \quad h=0, \ldots, k-1\end{cases}
$$

admits a unique solution $u \in C_{b}^{k, \alpha}$. Moreover, for any $t>b$,

$$
u(t)=\frac{1}{\Gamma(\alpha)} \int_{b}^{t} g(\tau)(t-\tau)^{\alpha-1} d \tau
$$

Proof. Let us start by proving the uniqueness claim. For this, let $u_{1}$ and $u_{2} \in C_{b}^{k, \alpha}$ be two different solutions of (2.7), and let $u:=u_{1}-u_{2}$. Then $u \in C_{b}^{k, \alpha}$ and

$$
\begin{cases}D_{b}^{\alpha} u(t)=0 & \text { in } \quad(b,+\infty) \\ u^{(h)}(b)=0 & \text { for any } h=0, \ldots, k-1\end{cases}
$$

Hence, recalling (1.2), for every $s>t$ we have that

$$
0=\Gamma(k-\alpha) \int_{b}^{s} D_{b}^{\alpha} u(t)(s-t)^{\alpha-k} d t=\int_{b}^{s}\left[\int_{b}^{t} \frac{u^{(k)}(\tau)}{(t-\tau)^{\alpha-k+1}} d \tau\right](s-t)^{\alpha-k} d t
$$

We recall the Euler's Beta-Function, defined, for $x$ and $y>0$, as

$$
\mathrm{B}(x, y):=\int_{0}^{1} \vartheta^{x-1}(1-\vartheta)^{y-1} d \vartheta
$$

and the fact that

$$
\mathrm{B}(x, y)=\mathrm{B}(y, x)=\frac{\Gamma(x) \Gamma(y)}{\Gamma(x+y)}
$$

see e.g. [AS64].

We employ the change of variable $\vartheta:=\frac{t-\tau}{s-\tau}$ and we observe that, for all $x, y>0$,

$$
\int_{\tau}^{s}(t-\tau)^{x-1}(s-t)^{y-1} d t=(s-\tau)^{x+y-1} \mathrm{~B}(x, y)
$$

and therefore

$$
\int_{\tau}^{s}(t-\tau)^{k-\alpha-1}(s-t)^{\alpha-k} d t=\mathrm{B}(k-\alpha, \alpha-k+1)=\Gamma(k-\alpha) \Gamma(\alpha-k+1) .
$$


Then,

$$
\begin{aligned}
\Gamma(k & -\alpha) \Gamma(\alpha-k+1)\left\|u^{(k)}\right\|_{L^{1}((b, s))}=\Gamma(k-\alpha) \Gamma(\alpha-k+1) \int_{b}^{s}\left|u^{(k)}(\tau)\right| d \tau \\
& =\int_{b}^{s}\left[\int_{\tau}^{s}(t-\tau)^{k-\alpha-1}(s-t)^{\alpha-k} d t\right]\left|u^{(k)}(\tau)\right| d \tau .
\end{aligned}
$$

As a result, by Fubini-Tonelli's Theorem,

$$
\begin{aligned}
\Gamma(k- & \alpha) \Gamma(\alpha-k+1)\left(u^{(k-1)}(s)-u^{(k-1)}(b)\right)=\Gamma(k-\alpha) \Gamma(\alpha-k+1) \int_{b}^{s} u^{(k)}(\tau) d \tau \\
& =\int_{b}^{s}\left[\int_{\tau}^{s}(t-\tau)^{k-\alpha-1}(s-t)^{\alpha-k} d t\right] u^{(k)}(\tau) d \tau \\
& =\int_{b}^{s}\left[\int_{b}^{t}(t-\tau)^{k-\alpha-1} u^{(k)}(\tau) d \tau\right](s-t)^{\alpha-k} d t .
\end{aligned}
$$

The latter term vanishes, in the light of (2.10), and therefore we conclude that

$$
u^{(k-1)}(s)-u^{(k-1)}(b)=0 .
$$

Recalling the initial condition in (2.9), we thereby obtain that $u^{(k-1)}(s)=0$. Since this is valid for all $s>b$, we have that $u^{(k-1)}$ vanishes identically in $[b,+\infty)$.

This in turn implies that $u^{(k-2)}$ is constant in $[b,+\infty)$. Recalling the initial condition in (2.9), we thus deduce that $u^{(k-2)}$ vanishes identically in $[b,+\infty)$.

Iterating this argument, we find that $u$ vanishes identically in $[b,+\infty)$, and therefore $u_{1}$ coincides with $u_{2}$ and the uniqueness claim in Lemma 2.3 is established.

To complete the proof of Lemma 2.3, it remains to check that if $u$ is defined as in (2.8), then $u \in C_{b}^{k, \alpha}$ and it satisfies (2.7). To check these facts, we first recall Lemma 2.2, according to which $u \in A C^{k-1}([b,+\infty))$, and, for any $t>b$,

$$
\begin{aligned}
u^{(k)}(t)=\frac{1}{\Gamma(\alpha)} & {\left[\int_{b}^{t} g^{(k)}(\tau)(t-\tau)^{\alpha-1} d \tau\right.} \\
& \left.+\sum_{i=0}^{k-1} \frac{(\alpha+i) \ldots(\alpha+i-k+1) g^{(i)}(b)}{\alpha(\alpha+1) \ldots(\alpha+i)}(t-b)^{\alpha+i-k}\right] .
\end{aligned}
$$

Therefore, in the notation of (1.3),

$$
\begin{aligned}
\Theta_{k, \alpha, u, t}(\sigma)= & u^{(k)}(\sigma)(t-\sigma)^{k-\alpha-1} \\
= & \frac{1}{\Gamma(\alpha)}\left[\int_{b}^{\sigma} g^{(k)}(\tau)(\sigma-\tau)^{\alpha-1}(t-\sigma)^{k-\alpha-1} d \tau\right. \\
& \left.\quad+\sum_{i=0}^{k-1} \frac{(\alpha+i) \ldots(\alpha+i-k+1) g^{(i)}(b)}{\alpha(\alpha+1) \ldots(\alpha+i)}(\sigma-b)^{\alpha+i-k}(t-\sigma)^{k-\alpha-1}\right] .
\end{aligned}
$$


We observe that

$$
\begin{aligned}
& \int_{b}^{t}\left|\sum_{i=0}^{k-1} \frac{(\alpha+i) \ldots(\alpha+i-k+1) g^{(i)}(b)}{\alpha(\alpha+1) \ldots(\alpha+i)}(\sigma-b)^{\alpha+i-k}(t-\sigma)^{k-\alpha-1}\right| d \sigma \\
\leq & \sum_{i=0}^{k-1}\left|\frac{(\alpha+i) \ldots(\alpha+i-k+1) g^{(i)}(b)}{\alpha(\alpha+1) \ldots(\alpha+i)}\right| \int_{b}^{t}(\sigma-b)^{\alpha+i-k}(t-\sigma)^{k-\alpha-1} d \sigma \\
= & \sum_{i=0}^{k-1}\left|\frac{(\alpha+i) \ldots(\alpha+i-k+1) g^{(i)}(b)}{\alpha(\alpha+1) \ldots(\alpha+i)}\right| \mathrm{B}(\alpha+i-k+1, k-\alpha)(t-b)^{i},
\end{aligned}
$$

where (2.12) has been used in the last line (with $x:=\alpha+i-k+1$ and $y:=k-\alpha$ ).

On the other hand, making again use of (2.12) with $x:=\alpha$ and $y:=k-\alpha$ here, we see that

$$
\begin{aligned}
& \int_{b}^{t}\left|\int_{b}^{\sigma} g^{(k)}(\tau)(\sigma-\tau)^{\alpha-1}(t-\sigma)^{k-\alpha-1} d \tau\right| d \sigma \\
\leq & \int_{b}^{t}\left[\int_{b}^{\sigma}\left|g^{(k)}(\tau)\right|(\sigma-\tau)^{\alpha-1}(t-\sigma)^{k-\alpha-1} d \tau\right] d \sigma \\
\leq & \int_{b}^{t}\left|g^{(k)}(\tau)\right|\left[\int_{\tau}^{t}(\sigma-\tau)^{\alpha-1}(t-\sigma)^{k-\alpha-1} d \sigma\right] d \tau \\
= & \mathrm{B}(\alpha, k-\alpha) \int_{b}^{t}\left|g^{(k)}(\tau)\right|(t-\tau)^{k-1} d \tau,
\end{aligned}
$$

which is finite, thanks to our assumptions on $g$.

Plugging this estimate and (2.14) into (2.13), we thereby deduce that, for all $t>b$,

$$
\int_{b}^{t}\left|\Theta_{k, \alpha, u, t}(\sigma)\right| d \sigma<+\infty
$$

and therefore $u \in C_{b}^{k, \alpha}$.

With this, it only remains to check (2.7). To this end, we observe that the initial point conditions are satisfied, due to (2.6). Moreover, (2.2) gives that

$$
\begin{aligned}
\Gamma(\alpha) u^{(k)}(t)= & \int_{b}^{t} g^{(k)}(\sigma)(t-\sigma)^{\alpha-1} d \sigma \\
& \quad+\sum_{i=0}^{k-1} \frac{(\alpha+i) \ldots(\alpha+i-k+1) g^{(i)}(b)}{\alpha(\alpha+1) \ldots(\alpha+i)}(t-b)^{\alpha+i-k},
\end{aligned}
$$


and therefore, in view of (1.2) and (2.12),

$$
\begin{aligned}
& \Gamma(\alpha) \Gamma(k-\alpha) D_{b}^{\alpha} u(t) \\
= & \Gamma(\alpha) \int_{b}^{t} \frac{u^{(k)}(\tau)}{(t-\tau)^{\alpha-k+1}} d \tau \\
= & \int_{b}^{t}\left(\int_{b}^{\tau} g^{(k)}(\sigma)(\tau-\sigma)^{\alpha-1} d \sigma\right)(t-\tau)^{k-\alpha-1} d \tau \\
& \quad+\sum_{i=0}^{k-1} \int_{b}^{t} \frac{(\alpha+i) \ldots(\alpha+i-k+1) g^{(i)}(b)}{\alpha(\alpha+1) \ldots(\alpha+i)}(\tau-b)^{\alpha+i-k}(t-\tau)^{k-\alpha-1} d \tau \\
= & \int_{b}^{t} g^{(k)}(\sigma)\left(\int_{\sigma}^{t}(\tau-\sigma)^{\alpha-1}(t-\tau)^{k-\alpha-1} d \tau\right) d \sigma \\
& \quad+\sum_{i=0}^{k-1} \mathrm{~B}(\alpha+i-k+1, k-\alpha) \frac{(\alpha+i) \ldots(\alpha+i-k+1) g^{(i)}(b)}{\alpha(\alpha+1) \ldots(\alpha+i)}(t-b)^{i} \\
= & \mathrm{B}(\alpha, k-\alpha) \int_{b}^{t} g^{(k)}(\sigma)(t-\sigma)^{k-1} d \sigma \\
& \quad+\sum_{i=0}^{k-1} \mathrm{~B}(\alpha+i-k+1, k-\alpha) \frac{(\alpha+i) \ldots(\alpha+i-k+1) g^{(i)}(b)}{\alpha(\alpha+1) \ldots(\alpha+i)}(t-b)^{i} .
\end{aligned}
$$

Hence, recalling (2.11) and using the fact that $\Gamma(z+1)=z \Gamma(z)$, we have that

$$
\begin{aligned}
& \Gamma(\alpha) \Gamma(k-\alpha) D_{b}^{\alpha} u(t) \\
= & \frac{\Gamma(\alpha) \Gamma(k-\alpha)}{(k-1) !} \int_{b}^{t} g^{(k)}(\sigma)(t-\sigma)^{k-1} d \sigma \\
& \quad+\sum_{i=0}^{k-1} \frac{\Gamma(\alpha+i-k+1) \Gamma(k-\alpha)}{i !} \cdot \frac{(\alpha+i) \ldots(\alpha+i-k+1) g^{(i)}(b)}{\alpha(\alpha+1) \ldots(\alpha+i)}(t-b)^{i} \\
= & \frac{\Gamma(\alpha) \Gamma(k-\alpha)}{(k-1) !} \int_{b}^{t} g^{(k)}(\sigma)(t-\sigma)^{k-1} d \sigma+\Gamma(\alpha) \Gamma(k-\alpha) \sum_{i=0}^{k-1} \frac{g^{(i)}(b)}{i !}(t-b)^{i} .
\end{aligned}
$$

As a consequence, recalling (2.3),

$$
\Gamma(\alpha) \Gamma(k-\alpha) D_{b}^{\alpha} u(t)=\Gamma(\alpha) \Gamma(k-\alpha) g(t),
$$

which gives that $D_{b}^{\alpha} u(t)=g(t)$, as desired.

A bootstrap regularity theory for time-fractional equations leads to additional smoothness of the solution. In our framework, the result needed is the following:

Lemma 2.4. Let $g \in C^{h}([b,+\infty))$ for every $h \in \mathbb{N}_{0}$, and $u \in C_{b}^{k, \alpha}$ be a solution of

$$
\left\{\begin{array}{l}
D_{b}^{\alpha} u(t)=g(t) \quad \text { in } \quad(b,+\infty), \\
u^{(h)}(b)=0 \quad \text { for any } \quad h=0, \ldots, k-1,
\end{array}\right.
$$

Then $u \in C^{\infty}((b,+\infty))$.

Proof. In light of (2.8), we can write, for every $t>b$,

$$
u(t)=\frac{1}{\Gamma(\alpha)} \int_{0}^{t-b} g(t-\sigma) \sigma^{\alpha-1} d \sigma .
$$

The desired result follows by taking derivatives in $t$. 


\subsection{Existence of a sequence of Caputo-stationary functions that tends to the function $t^{\alpha}$}

Now, we generalize some results contained in Section 3 of [Buc17] concerning the boundary asymptotics of solutions of fractional equations, and we construct a sequence of Caputostationary functions which tends to the function $t^{\alpha}$ uniformly on bounded subintervals of $(0,+\infty)$. Differently from the previous literature, we deal with fractional derivatives of any order.

More precisely, the result that we need is the following:

Lemma 2.5. Let $b \in(-\infty, 0] \cup\{-\infty\}$. Let $\psi_{0} \in C_{-\infty}^{k, \alpha}$ be such that

$$
\psi_{0}^{(k)}=0 \quad \text { for any } \quad t \in(-\infty, 0), \quad \text { and } \quad \psi_{0}(t)=0 \quad \text { for any } \quad t \in\left[\frac{3}{4}, 1\right] \text {. }
$$

Then, there exists $\psi \in C_{b}^{k, \alpha}$ such that

$$
\left\{\begin{array}{lll}
D_{b}^{\alpha} \psi(t)=0 & \text { in } & (1,+\infty) \\
\psi(t)=\psi_{0}(t) & \text { in } & (-\infty, 1]
\end{array}\right.
$$

Moreover, $\psi \in C^{\infty}((1,+\infty))$, and we have that

$$
\psi(1+\epsilon)=\kappa \epsilon^{\alpha}+o\left(\epsilon^{\alpha}\right)
$$

as $\epsilon \rightarrow 0^{+}$, with

$$
\kappa:=-\frac{1}{\Gamma(\alpha) \Gamma(k-\alpha)} \int_{0}^{1}\left(\int_{0}^{3 / 4} \psi_{0}^{(k)}(\omega)(1-\omega)^{k-\alpha-1} d \omega\right)(1-z)^{\alpha-1} d z \in \mathbb{R} .
$$

Proof. For every $t \in[1,+\infty)$, we set

$$
g(t):=-\frac{1}{\Gamma(k-\alpha)} \int_{0}^{3 / 4} \psi_{0}^{(k)}(\tau)(t-\tau)^{k-\alpha-1} d \tau
$$

By construction, the term $t-\tau$ in the integrand above never vanishes, thus permitting to take derivatives inside the integral sign. Consequently, we have that $g \in C^{h}([1,+\infty))$ for every $h \in \mathbb{N}_{0}$.

For any $t>1$, we define

$$
\psi(t):=\frac{1}{\Gamma(\alpha)} \int_{1}^{t} g(\tau)(t-\tau)^{\alpha-1} d \tau .
$$

We know from Lemma 2.3 that

$$
\psi \in C_{1}^{k, \alpha}
$$

is a solution of

$$
\begin{cases}D_{1}^{\alpha} \psi(t)=g(t) & \text { in } \quad(1,+\infty) \\ \psi^{(h)}(1)=0 & \text { for any } \quad h=0, \ldots, k-1\end{cases}
$$

and also $\psi \in C^{\infty}((1,+\infty))$, due to Lemma 2.4. 
We also extend $\psi$ in $(-\infty, 1]$ by setting $\psi(t):=\psi_{0}(t)$ for all $t \in(-\infty, 1]$. Since $\psi_{0}$ vanishes in $\left[\frac{3}{4}, 1\right]$, using the initial condition in $(2.22)$ we have that $\psi_{0}^{(j)}(1)=0=\psi^{(j)}(1)$ for each $j \in\{1, \ldots, k-1\}$. Thus, recalling (2.21) and using Lemma A.2 (exploited here with $f:=\psi_{0}$ and $g:=\psi$ ), we find that

$$
\psi \in C_{b}^{k, \alpha}
$$

Then, in light of (2.22), we can write that

$$
\begin{cases}D_{1}^{\alpha} \psi(t)=g(t) & \text { in }(1,+\infty) \\ \psi=\psi_{0} & \text { in }(-\infty, 1]\end{cases}
$$

From (2.23), it follows in particular that $\psi \in C_{0}^{k, \alpha}$. Consequently, by (2.24) and Lemma 2.1, we have that

$$
\psi \text { is a solution of }(2.16) \text { with } b=0 \text {. }
$$

Since $\psi(t)=\psi_{0}(t)=\psi_{0}(0)$ if $t \in(-\infty, 0]$, we see that $\psi^{(k)}(\tau)=0$ in $(-\infty, 0)$ and therefore, for every $t \in(1,+\infty)$,

$$
D_{b}^{\alpha} \psi(t)=\frac{1}{\Gamma(k-\alpha)} \int_{b}^{t} \frac{\psi^{(k)}(\tau)}{(t-\tau)^{\alpha-k+1}} d \tau=\frac{1}{\Gamma(k-\alpha)} \int_{0}^{t} \frac{\psi^{(k)}(\tau)}{(t-\tau)^{\alpha-k+1}} d \tau=D_{0}^{\alpha} \psi(t)=0
$$

thanks to (2.25) and this gives (2.16) (alternatively, one can use Lemma A.3 here).

Hence, to complete the proof of Lemma 2.5, it only remains to establish (2.17). For this, let $\epsilon>0$ and $t:=1+\epsilon$. Then, by (2.20),

$$
\Gamma(\alpha) \psi(1+\epsilon)=\int_{1}^{1+\epsilon} g(\tau)(1+\epsilon-\tau)^{\alpha-1} d \tau=\epsilon^{\alpha} \int_{0}^{1} g(\epsilon z+1)(1-z)^{\alpha-1} d z,
$$

where the change of variables $\tau=\epsilon z+1$ has been used.

Furthermore, by (2.19),

$$
g(\epsilon z+1)=-\frac{1}{\Gamma(k-\alpha)} \int_{0}^{3 / 4} \psi_{0}^{(k)}(\omega)(\epsilon z+1-\omega)^{k-\alpha-1} d \omega
$$

Hence

$$
\Gamma(\alpha) \psi(1+\epsilon)=-\frac{\epsilon^{\alpha}}{\Gamma(k-\alpha)} \int_{0}^{1}\left(\int_{0}^{3 / 4} \psi_{0}^{(k)}(\omega)(\epsilon z+1-\omega)^{k-\alpha-1} d \omega\right)(1-z)^{\alpha-1} d z .
$$

This gives that

$$
\lim _{\epsilon \rightarrow 0^{+}} \epsilon^{-\alpha} \Gamma(\alpha) \Gamma(k-\alpha) \psi(1+\epsilon)=-\int_{0}^{1}\left(\int_{0}^{3 / 4} \psi_{0}^{(k)}(\omega)(1-\omega)^{k-\alpha-1} d \omega\right)(1-z)^{\alpha-1} d z .
$$

This, together with (2.18), establishes (2.17), as desired.

In our setting, it is crucial that we can choose $\psi_{0}$ such that $\kappa$ in (2.18) is not zero. This is warranted by the following observation: 
Lemma 2.6. There exists $\psi_{0}$ satisfying all the assumptions of Lemma 2.5 and such that $\kappa>$ 0 , where the setting in (2.18) has been used.

Proof. We let

$$
\psi_{0}(t):= \begin{cases}(-1)^{k-1} \sum_{j=0}^{k-1}\left(\begin{array}{l}
k \\
j
\end{array}\right)\left(\frac{3}{4}\right)^{k-j} t^{j} & \text { if } t \leq 0 \\
(-1)^{k-1}\left(\frac{3}{4}-t\right)^{k} & \text { if } t \in\left(0, \frac{3}{4}\right), \\
0 & \text { if } t \geq \frac{3}{4} .\end{cases}
$$

We observe that the statements in (2.15) are satisfied in this case. Furthermore, we claim that

$$
\psi_{0} \in C_{-\infty}^{k, \alpha}
$$

Indeed, using Lemma A.2 with

$$
f(t):=(-1)^{k-1}\left(\frac{3}{4}-t\right)^{k}
$$

$g:=0, a:=0$ and $b:=\frac{3}{4}$, we obtain that $\tilde{\psi}_{0} \in C_{0}^{k, \alpha}$, where

$$
\tilde{\psi}_{0}(t):= \begin{cases}(-1)^{k-1}\left(\frac{3}{4}-t\right)^{k} & \text { if } t \in\left(0, \frac{3}{4}\right), \\ 0 & \text { if } t \geq \frac{3}{4} .\end{cases}
$$

Then, using again Lemma A.2 with

$$
f(t):=(-1)^{k-1} \sum_{j=0}^{k-1}\left(\begin{array}{l}
k \\
j
\end{array}\right)\left(\frac{3}{4}\right)^{k-j} t^{j}=(-1)^{k-1}\left(\frac{3}{4}-t\right)^{k}-(-1)^{k-1} t^{k}
$$

$g:=\tilde{\psi}_{0}, a:=-\infty$ and $b:=0$, we obtain (2.26), as desired.

We also notice that, for any $t \in\left(0, \frac{3}{4}\right)$, we have that

$$
\psi_{0}^{(k)}(t)=-k !<0
$$

and so, recalling (2.18), we have that $\kappa>0$.

Now we point out that the function built in Lemmata 2.5 and 2.6 can be conveniently rescaled, taking advantage of the scaling invariance of the operator, and in this way one can single out the boundary behavior. Namely, we have that:

Lemma 2.7. There exists a sequence $\left(v_{j}\right)_{j \in \mathbb{N}}$ of functions $v_{j} \in C_{-\infty}^{k, \alpha} \cap C^{\infty}((0,+\infty))$ such that, for any $j \in \mathbb{N}$, $v_{j}$ solves the following problem

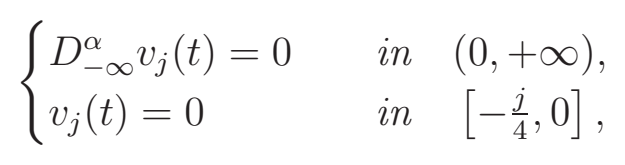


and, for any $t>0$,

$$
\lim _{j \rightarrow+\infty} v_{j}(t)=\kappa t^{\alpha},
$$

for some $\kappa>0$, and the convergence is uniform on any bounded subinterval of $(0,+\infty)$.

In addition,

$$
v_{j}^{(k)}=0 \text { in }(-\infty,-j) .
$$

Proof. Let $\psi$ be the function given in Lemmata 2.5 and 2.6, used here with $b:=-\infty$, and define for any $j \in \mathbb{N}$

$$
v_{j}(t):=j^{\alpha} \psi\left(\frac{t}{j}+1\right) .
$$

Since $\psi \in C_{-\infty}^{k, \alpha} \cap C^{\infty}((1,+\infty))$, we have that $v_{j} \in C_{-\infty}^{k, \alpha} \cap C^{\infty}((0,+\infty))$.

We claim that for any $j \in \mathbb{N}, v_{j}$ solves (2.27). Indeed, recalling (2.15) and (2.16), for any $t \in\left[-\frac{j}{4}, 0\right]$ we have that $\frac{t}{j}+1 \in\left[\frac{3}{4}, 1\right]$, and

$$
v_{j}(t)=j^{\alpha} \psi\left(\frac{t}{j}+1\right)=j^{\alpha} \psi_{0}\left(\frac{t}{j}+1\right)=0 .
$$

Moreover, if $t>0$, we have that $\frac{t}{j}+1>1$ and therefore, using the change of variables $y:=$ $\frac{\tau}{j}+1$ and $(2.16)$,

$$
\begin{aligned}
D_{-\infty}^{\alpha} v_{j}(t) & =\frac{1}{\Gamma(k-\alpha)} \int_{-\infty}^{t} v_{j}^{(k)}(\tau)(t-\tau)^{k-\alpha-1} d \tau \\
& =\frac{j^{\alpha-k}}{\Gamma(k-\alpha)} \int_{-\infty}^{t} \psi^{(k)}\left(\frac{\tau}{j}+1\right)(t-\tau)^{k-\alpha-1} d \tau \\
& =\frac{j^{\alpha-k+1}}{\Gamma(k-\alpha)} \int_{-\infty}^{\frac{t}{j}+1} \psi^{(k)}(y)(t-j(y-1))^{k-\alpha-1} d y \\
& =\frac{1}{\Gamma(k-\alpha)} \int_{-\infty}^{\frac{t}{j}+1} \psi^{(k)}(y)\left(\frac{t}{j}+1-y\right)^{k-\alpha-1} d y \\
& =D_{-\infty}^{\alpha} \psi\left(\frac{t}{j}+1\right) \\
& =0
\end{aligned}
$$

This proves (2.27).

Now, let $I$ be a bounded subinterval of $(0,+\infty)$. Using formula (2.17), for $t>0$ and for large $j$, we have that

$$
\begin{aligned}
& \sup _{t \in I}\left|v_{j}(t)-\kappa t^{\alpha}\right|=\sup _{t \in I}\left|j^{\alpha} \psi\left(\frac{t}{j}+1\right)-\kappa t^{\alpha}\right|=\sup _{t \in I}\left|j^{\alpha}\left(\kappa \frac{t^{\alpha}}{j^{\alpha}}+o\left(\frac{t^{\alpha}}{j^{\alpha}}\right)\right)-\kappa t^{\alpha}\right| \\
& =\sup _{t \in I} j^{\alpha} o\left(\frac{t^{\alpha}}{j^{\alpha}}\right)=j^{\alpha} o\left(\frac{1}{j^{\alpha}}\right)=o(1),
\end{aligned}
$$

thus proving the desired asymptotics in (2.28), and $\kappa>0$ here in view of Lemma 2.6.

Finally, recalling (2.15) and (2.16), we have that if $t<-j$

$$
v_{j}^{(k)}(t)=j^{\alpha-k} \psi^{(k)}\left(\frac{t}{j}+1\right)=j^{\alpha-k} \psi_{0}^{(k)}\left(\frac{t}{j}+1\right)=0,
$$

and this proves (2.29). 


\subsection{Maximal span property and proof of Theorem 1.1}

We now exploit a method introduced in [DSV17] and we take advantage of the boundary asymptotics established in Lemma 2.6 to construct solutions of linear time-fractional equations with a prescribed jet of derivatives at a point. From this, the proof of Theorem 1.1 will be completed, by polynomial approximation and scaling. In our strategy is also technically more convenient to prove a slightly different modified version of Theorem 1.1, in which the initial point in which the Caputo derivative is $-\infty$ and the approximating function is constant near $-\infty$. Namely, we prove the following result:

Theorem 2.8. Let $h \in \mathbb{N}_{0}, k \in \mathbb{N}$, and $\alpha \in(k-1, k)$.

Then, for every $f \in C^{h}([0,1])$ and $\epsilon>0$, there exist $a<0$ and $u \in C_{-\infty}^{k, \alpha}$ such that

$$
\begin{array}{ll} 
& D_{-\infty}^{\alpha} u(t)=0 \quad \text { in } \quad[0,+\infty) \\
& u^{(k)}=0 \quad \text { for all } t \in(-\infty, a] \\
\text { and } \quad & \|u-f\|_{C^{h}([0,1])}<\epsilon .
\end{array}
$$

By Lemma A.1, we observe that

Theorem 2.8 implies Theorem 1.1.

Hence, in light of (2.33), to prove Theorem 1.1 we will focus on the proof of Theorem 2.8. For this, one of the crucial arguments is given by the following "cherry picking" result:

Proposition 2.9. For any $m \in \mathbb{N}$, there exist $p>0, R>0$, and $v \in C_{-\infty}^{k, \alpha} \cap C^{\infty}((0,+\infty))$ such that

$$
\begin{aligned}
& \left\{\begin{array}{l}
D_{-\infty}^{\alpha} v(t)=0 \\
v^{(k)}=0
\end{array} \quad \text { for all } t \in(0,+\infty),\right. \\
& v^{(l)}(p)=0 \quad \text { for any } l \in\{0, \ldots, m-1\},
\end{aligned}
$$

and

$$
v^{(m)}(p)=1
$$

Proof. Let $\mathcal{Z}_{0}$ be the set containing all the functions $v \in C_{-\infty}^{k, \alpha} \cap C^{\infty}((0,+\infty))$ such that

$$
D_{-\infty}^{\alpha} v=0 \text { in }(0,+\infty),
$$

and for which there exists $R>0$ such that

$$
v^{(k)}=0 \quad \text { in }(-\infty,-R) .
$$

Let also $\mathcal{Z}:=\mathcal{Z}_{0} \times(0,+\infty)$.

To each pair $(v, t) \in \mathcal{Z}$ we associate the vector $\left(v(t), v^{\prime}(t), \ldots, v^{(m)}(t)\right) \in \mathbb{R}^{m+1}$ and consider $\mathcal{V}$ to be the set

$$
\mathcal{V}:=\left\{\left(v(t), v^{\prime}(t), \ldots, v^{(m)}(t)\right), \quad \text { with }(v, t) \in \mathcal{Z}\right\} .
$$

We point out that

$$
\mathcal{V} \text { is a vector space. }
$$


Notice indeed that if $\left(v_{1}, t\right),\left(v_{2}, t\right) \in \mathcal{Z}$ and $\lambda_{1}, \lambda_{2} \in \mathbb{R}$, we have that $v_{i} \in C_{-\infty}^{k, \alpha} \cap$ $C^{\infty}((0,+\infty))$, and therefore $v_{*}:=\lambda_{1} v_{1}+\lambda_{2} v_{2} \in C_{-\infty}^{k, \alpha} \cap C^{\infty}((0,+\infty))$. Also, $v_{*}$ satisfies $(2.35)$ by linearity of the operator $D_{-\infty}^{\alpha}$. In addition, by $(2.36)$, for each $i \in\{1,2\}$ we know that $v_{i}^{(k)}=0$ in $\left(-\infty,-R_{i}\right)$ for some $R_{i}>0$, and therefore $v_{*}$ satisfies $(2.36)$ with $R:=\max \left\{R_{1}, R_{2}\right\}>0$. This completes the proof of (2.38).

Now, we claim that

$$
\mathcal{V}=\mathbb{R}^{m+1}
$$

To check this, we suppose by contradiction that $\mathcal{V}$ lies in a proper subspace of $\mathbb{R}^{m+1}$. Then, by $(2.38), \mathcal{V}$ must lie in a hyperplane, hence there exists

$$
\left(c_{0}, \ldots, c_{m}\right) \in \mathbb{R}^{m+1} \backslash\{0\}
$$

which is orthogonal to any vector $\left(v(t), \ldots, v^{(m)}(t)\right)$ with $(v, t) \in \mathcal{Z}$, namely

$$
\sum_{i=0}^{m} c_{i} v^{(i)}(t)=0
$$

We notice that for any $j \geq 1$ the pair $\left(v_{j}, t\right)$, with $v_{j}$ satisfying (2.27) and (2.29), and $t \in(0,+\infty)$, belongs to $\mathcal{Z}$. Consequently, writing (2.41) in this case, it follows that, for any $j \geq 1$

$$
\sum_{i=0}^{m} c_{i} v_{j}^{(i)}(t)=0
$$

Let now $\varphi \in C_{c}^{\infty}((0,+\infty))$. Integrating by parts, by Lemma 2.7 and the Dominated Convergence Theorem, we have that, for any $i \in \mathbb{N}$,

$$
\begin{gathered}
\lim _{j \rightarrow+\infty} \int_{-\infty}^{+\infty} v_{j}^{(i)}(t) \varphi(t) d t=(-1)^{i} \lim _{j \rightarrow+\infty} \int_{-\infty}^{+\infty} v_{j}(t) \varphi^{(i)}(t) d t \\
=(-1)^{i} \int_{-\infty}^{+\infty} \kappa t^{\alpha} \varphi^{(i)}(t) d t=\kappa \int_{-\infty}^{+\infty}\left(t^{\alpha}\right)^{(i)} \varphi(t) d t
\end{gathered}
$$

Multiplying by $c_{i}$ and summing up, recalling also (2.42), we thereby obtain that

$$
0=\lim _{j \rightarrow+\infty} \int_{-\infty}^{+\infty} \sum_{i=0}^{m} c_{i} v_{j}^{(i)}(t) \varphi(t) d t=\kappa \int_{-\infty}^{+\infty} \sum_{i=0}^{m} c_{i}\left(t^{\alpha}\right)^{(i)} \varphi(t) d t
$$

for any $\varphi \in C_{c}^{\infty}((0,+\infty))$.

This gives that, for every $t \in(0,+\infty)$,

$$
0=\kappa \sum_{i=0}^{m} c_{i}\left(t^{\alpha}\right)^{(i)}=\kappa \sum_{i=0}^{m} c_{i} \alpha(\alpha-1) \ldots(\alpha-i+1) t^{\alpha-i} .
$$

Then, we divide this relation by $\kappa>0$ and multiply by $t^{m-\alpha}$ : in this way we obtain that, for every $t \in(0,+\infty)$,

$$
\sum_{i=0}^{m} c_{i} \alpha(\alpha-1) \ldots(\alpha-i+1) t^{m-i}=0
$$


The identity above describes a polynomial which vanishes for any $t \in(0,+\infty)$. As a result, the Identity Principle for polynomials leads to

$$
c_{i} \alpha(\alpha-1) \ldots(\alpha-i+1)=0,
$$

for all $i \in\{0, \ldots, m\}$.

Consequently, since $\alpha \in \mathbb{R} \backslash \mathbb{N}$, the product $\alpha(\alpha-1) \ldots(\alpha-i+1)$ never vanishes, and so the coefficients $c_{i}$ are forced to be null for any $i \in\{0, \ldots, m\}$. This is in contradiction with (2.40), and therefore the proof of (2.39) is complete.

From this, the desired claim in Proposition 2.9 plainly follows.

Now, we use the function constructed in Proposition 2.9 to prove Theorem 1.1.

Proof of Theorem 1.1. In light of (2.33), we focus on the proof of Theorem 2.8. Also, it is sufficient to prove Theorem 2.8 for monomials: indeed, once proved in this case, the claim is true for polynomials simply by linearity of the operator $D_{-\infty}^{\alpha}$, and then it is also true for smooth functions, by exploiting the density of polynomials in the space $C^{h}([0,1])$ given by the Stone-Weierstraß Theorem.

Therefore, in place of the generic function $f$ in Theorem 2.8, we can consider the monomial $q_{m}(t):=\frac{t^{m}}{m !}$. We take $p, R>0$, and $v$ as in Proposition 2.9. Also, let

$$
\delta \in(0,1)
$$

to be chosen conveniently in the sequel.

Let $u$ be the function

$$
u(t):=\frac{v(\delta t+p)}{\delta^{m}} .
$$

By construction, we have that $u \in C_{-\infty}^{k, \alpha} \cap C^{\infty}\left(\left(-\frac{p}{\delta},+\infty\right)\right)$, and, for any $t \in\left(-\frac{p}{\delta},+\infty\right)$,

$$
\begin{aligned}
\Gamma(k-\alpha) D_{-\infty}^{\alpha} u(t) & =\int_{-\infty}^{t} u^{(k)}(\tau)(t-\tau)^{k-\alpha-1} d \tau \\
& =\delta^{k-m} \int_{-\infty}^{t} v^{(k)}(\delta \tau+p)(t-\tau)^{k-\alpha-1} d \tau \\
& =\delta^{k-m} \delta^{\alpha-k+1} \int_{-\infty}^{\delta t+p} v^{(k)}(y)(\delta t+p-y)^{k-\alpha-1} \frac{d y}{\delta} \\
& =\delta^{\alpha-m} \Gamma(k-\alpha) D_{-\infty}^{\alpha} v(\delta t+p) \\
& =0 .
\end{aligned}
$$

Then, we have that $u \in C_{-\infty}^{k, \alpha}$, and since $-\frac{p}{\delta}<0$, we also see that $D_{-\infty}^{\alpha} u(t)=0$ in $[0,+\infty)$.

This proves (2.30). In addition, we see that (2.31) follows from (2.34) and (2.44) (taking $\left.a:=\frac{-R-p}{\delta}\right)$.

Hence, we now focus on the proof of (2.32). For this, we observe that

$$
u^{(l)}(0)=\delta^{l-m} v^{(l)}(p)=0, \quad \text { for any } l \in\{0, \ldots, m-1\}
$$

and

$$
u^{(m)}(0)=v^{(m)}(p)=1 .
$$

Now, for any $t>-\frac{p}{\delta}$, we set

$$
g(t):=u(t)-q_{m}(t)
$$


We have that

$$
\begin{aligned}
& g^{(l)}(0)=0, \quad \text { for any } l \in\{0, \ldots, m\}, \quad \text { and } \\
& g^{(m+l)}(t)=u^{(m+l)}(t)=\delta^{l} v^{(m+l)}(\delta t+p), \quad \text { for any } l \in\{1,2, \ldots\} \text {. }
\end{aligned}
$$

Hence, for all $t \in[0,1]$ and $l \in\{1,2, \ldots\}$,

$$
\left|g^{(m+l)}(t)\right| \leq \delta^{l} \sup _{y \in[p, p+\delta]}\left|v^{(m+l)}(y)\right|=\tilde{c} \delta^{l}
$$

where $\tilde{c}$ is a positive constant, depending on $v, m$ and $l$.

Now, we consider the derivative of order $j \in\{0, \ldots, h\}$ of $g$ (with the notation that the derivative of order zero coincides with the function itself), and we take its Taylor expansion with Lagrange remainder. In this way, in view of $(2.45)$, we have that, for every $t \in(0,1)$,

$$
g^{(j)}(t)=\sum_{i=\max \{j, m+1\}}^{j+m+1} g^{(i)}(0) \frac{t^{i-j}}{(i-j) !}+g^{(m+h+2)}(c) \frac{t^{m+2}}{(m+2) !},
$$

for some $c \in(0, t)$ possibly depending on $j, m$ and $t$.

As a consequence, using (2.43) and (2.46), and possibly renaming the constants, we obtain that, for any $t \in[0,1]$,

$$
\left|q_{m}^{(j)}(t)-u^{(j)}(t)\right|=\left|g^{(j)}(t)\right| \leq C \delta,
$$

with $C>0$ possibly depending on $j$ and $m$. By summing this inequality over $j \in\{1, \ldots, h\}$, and by choosing $\delta$ sufficiently small with respect to $\epsilon$, we complete the proof of $(2.32)$.

\section{Proof of Corollary 1.2}

The proof of Corollary 1.2 relies on Theorem 1.1 and on a change of variable induced by the function $\psi$. For this, we make the following observation:

Lemma 3.1. Let $a, \alpha$ and $\psi$ be as in (1.8). Then, for any $t>a$,

$$
D_{a}^{\alpha, \psi} u(t)=D_{\psi(a)}^{\alpha}\left(u \circ \psi^{-1}\right)(\psi(t))
$$

Proof. Using the change of variable $\omega:=\psi(\tau)$, we have that $\psi^{\prime}(\tau) d \tau=d \omega$, and $\frac{1}{\psi^{\prime}(\tau)} \frac{d}{d \tau}=\frac{d}{d \omega}$. Thus, from (1.8) we see that

$$
D_{a}^{\alpha, \psi} u(t)=\frac{1}{\Gamma(k-\alpha)} \int_{\psi(a)}^{\psi(t)} \frac{\left(u \circ \psi^{-1}\right)^{(k)}(\omega)}{(\psi(t)-\omega)^{\alpha-k+1}} d \omega=D_{\psi(a)}^{\alpha}\left(u \circ \psi^{-1}\right)(\psi(t)),
$$

and this proves (3.1).

Now we can complete the proof of Corollary 1.2.

Proof of Corollary 1.2. We claim that

$$
\text { for every } \lambda \in(-\infty, \psi(0)) \text { there exists } t_{\lambda} \in(-\infty, 0) \text { such that } \psi\left(t_{\lambda}\right)=\lambda \text {. }
$$


To check this, we exploit (1.9) to find $\theta_{\lambda}<0$ such that $\psi\left(\theta_{\lambda}\right)<\lambda$. Since $\psi(0)>\lambda$, the result in (3.2) follows from the Mean Value Theorem.

Now, for any $\omega \in[\psi(0), \psi(1)]$ we let $\tilde{f}(\omega):=f\left(\psi^{-1}(\omega)\right)$. Notice that $\tilde{f} \in C^{h}([\psi(0), \psi(1)])$. Hence, in light of Theorem 1.1, we find $\tilde{a} \in(-\infty, \psi(0))$ and $\tilde{u} \in C_{\tilde{a}}^{k, \alpha}$ such that

$$
\begin{array}{ll} 
& D_{\tilde{a}}^{\alpha} \tilde{u}(\omega)=0 \quad \text { for every } \omega \in[\psi(0),+\infty) \\
\text { and } \quad & \|\tilde{u}-\tilde{f}\|_{C^{h}([\psi(0), \psi(1)])}<\epsilon .
\end{array}
$$

In light of (3.2), there exists $a \in(-\infty, 0)$ such that

$$
\psi(a)=\tilde{a} .
$$

Then, we set, for any $t \in \mathbb{R}$ and $\omega \in[\psi(0), \psi(1)]$,

$$
\begin{aligned}
u(t) & :=\tilde{u}(\psi(t)) \\
\text { and } \quad \tilde{v}(\omega) & :=\tilde{u}(\omega)-\tilde{f}(\omega)=u\left(\psi^{-1}(\omega)\right)-f\left(\psi^{-1}(\omega)\right) .
\end{aligned}
$$

When $t \in[0,1]$, we also set

$$
v(t):=\tilde{v}(\psi(t))=u(t)-f(t) .
$$

By the Faà di Bruno Formula, for any $j \in\{0, \ldots, h\}$,

$$
v^{(j)}(t)=\frac{d^{j}}{d t^{j}} \tilde{v}(\psi(t))=j ! \sum_{m=1}^{j} \frac{\left(D^{m} \tilde{v}\right)(\psi(t))}{m !} \sum_{\substack{h_{1}, \cdots, h_{m} \geq 1 \\ h_{1}+\cdots+h_{m}=j}} \frac{D^{h_{1}} \psi(t)}{h_{1} !} \cdots \frac{D^{h_{m}} \psi(t)}{h_{m} !}
$$

and therefore

$$
\begin{aligned}
\| u- & f\left\|_{C^{h}([0,1])}=\right\| v \|_{C^{h}([0,1])}=\sum_{j=0}^{h} \sup _{t \in(0,1)}\left|v^{(j)}(t)\right| \\
& \leq h ! \sum_{j=0}^{h} \sum_{m=1}^{j} \sup _{t \in(0,1)}\left|\left(D^{m} \tilde{v}\right)(\psi(t))\right| \sum_{\substack{h_{1}, \cdots, h_{m} \geq 1 \\
h_{1}+\cdots+h_{m}=j}}\left|D^{h_{1}} \psi(t)\right| \cdots\left|D^{h_{m}} \psi(t)\right| \\
& \leq h !\|\tilde{v}\|_{C^{h}([\psi(0), \psi(1)])} \sum_{j=0}^{h} \sum_{m=1}^{j} \sum_{\substack{h_{1}, \cdots, h_{m} \geq 1 \\
h_{1}+\cdots+h_{m}=j}}\|\psi(t)\|_{C^{h}([0,1])}^{m} \\
& =C_{h, \psi}\|\tilde{v}\|_{C^{h}([\psi(0), \psi(1)])},
\end{aligned}
$$

for a suitable constant $C_{h, \psi}>0$.

As a result, we have that

$$
\|u-f\|_{C^{h}([0,1])} \leq C_{h, \psi}\|\tilde{u}-\tilde{f}\|_{C^{h}([\psi(0), \psi(1)])} \leq C_{h, \psi} \epsilon,
$$

which is the approximation estimate claimed in Corollary 1.2 (up to renaming $\epsilon$ ).

Furthermore, for any $t \geq 0$, we have that $\psi(t) \geq \psi(0)$ and, as a consequence, by Lemma 3.1 and (3.3),

$$
D_{a}^{\alpha, \psi} u(t)=D_{\psi(a)}^{\alpha}\left(u \circ \psi^{-1}\right)(\psi(t))=D_{\tilde{a}}^{\alpha} \tilde{u}(\psi(t))=0,
$$

thus completing the proof of Corollary 1.2. 


\section{A Caputo-stationary functions with vanishing $k$ th deriva- tives near $-\infty$}

In this appendix, we remark that Caputo-stationary functions with initial point $-\infty$ that have vanishing $k$ th derivative near $-\infty$ are also Caputo-stationary for a fixed point beyond its constancy interval. Namely, we have that:

Lemma A.1. Let $a \in \mathbb{R}$. Let $I \Subset(a,+\infty)$ be an interval. Let $k \in \mathbb{N}$ and $\alpha \in(k-1, k)$, and assume that $u \in C_{-\infty}^{k, \alpha}$, and that $u^{(k)}=0$ in $(-\infty, a)$.

Then,

$$
\begin{aligned}
& u \in C_{a}^{k, \alpha} \\
& \text { and } \quad D_{a}^{\alpha} u=D_{-\infty}^{\alpha} u \quad \text { in } I .
\end{aligned}
$$

Proof. By (1.4), we see that if $b \in(-\infty, a] \cup\{-\infty\}$, then $C_{b}^{k, \alpha} \subseteq C_{a}^{k, \alpha}$, and so (A.1) plainly follows. Furthermore, $u^{(k)}$ vanishes in $(-\infty, a)$, and consequently, for any $t \in I$,

$$
0=\int_{-\infty}^{t} \frac{u^{(k)}(\tau)}{(t-\tau)^{\alpha-k+1}} d \tau=\int_{a}^{t} \frac{u^{(k)}(\tau)}{(t-\tau)^{\alpha-k+1}} d \tau
$$

which proves (A.2).

A counterpart of Lemma A.1 allows us to extend a function with its Taylor polynomial maintaining its Caputo derivative. For this, we first point out that this operation is compatible with the functional setting in (1.4):

Lemma A.2. Let $a \in \mathbb{R} \cup\{-\infty\}$ and $b \in(a,+\infty)$. Let $k \in \mathbb{N}$ and $\alpha \in(k-1, k)$. Let $f \in C_{a}^{k, \alpha}, g \in C_{b}^{k, \alpha}$ and assume that

$$
f^{(j)}(b)=g^{(j)}(b) \quad \text { for all } j \in\{0, \ldots, k-1\} .
$$

Let

$$
\overline{(a,+\infty)} \ni t \mapsto h(t):= \begin{cases}f(t) & \text { if } t \in \overline{(a, b)}, \\ g(t) & \text { if } t \in(b,+\infty)\end{cases}
$$

Then $h \in C_{a}^{k, \alpha}$.

Proof. Since $f \in C^{k-1}(\overline{(a,+\infty)})$ and $g \in C^{k-1}([b,+\infty))$, we obtain from (A.3) that $h \in$ $C^{k-1}(\overline{(a,+\infty)})$, and, for every $t \in \overline{(a,+\infty)}$ and $j \in\{0, \ldots, k-1\}$,

$$
h^{(j)}(t)= \begin{cases}f^{(j)}(t) & \text { if } t \in \overline{(a, b)}, \\ g^{(j)}(t) & \text { if } t \in(b,+\infty) .\end{cases}
$$

In particular, we see from (A.3) that

$$
h^{(j)}(b)=f^{(j)}(b)=g^{(j)}(b), \quad \text { for all } j \in\{0, \ldots, k-1\} .
$$

Using that $f^{(j)} \in A C(\overline{(a, b)})$ for each $j \in\{0, \ldots, k-1\}$, we can write that, for every $t_{1}, t_{2} \in$ $\overline{(a, b)}$,

$$
f^{(j)}\left(t_{2}\right)-f^{(j)}\left(t_{1}\right)=\int_{t_{1}}^{t_{2}} F_{j}(\tau) d \tau
$$


for a suitable Lebesgue integrable function $F_{j}$.

Similarly, if $T>b$, since $g^{(j)} \in A C([b, T])$, we have that for every $t_{1}, t_{2} \in[b, T]$,

$$
g^{(j)}\left(t_{2}\right)-g^{(j)}\left(t_{1}\right)=\int_{t_{1}}^{t_{2}} G_{j}(\tau) d \tau,
$$

for a suitable Lebesgue integrable function $G_{j}$.

Then, given $T>b$, we define

$$
H_{j}(t)= \begin{cases}F_{j}(t) & \text { if } t \in \overline{(a, b)}, \\ G_{j}(t) & \text { if } t \in(b, T] .\end{cases}
$$

We have that $H_{j}$ is Lebesgue integrable and, if $t_{1} \in \overline{(a, b)}$ and $t_{2} \in(b, T]$, recalling (A.4) we see that

$$
\begin{aligned}
h^{(j)}\left(t_{2}\right)-h^{(j)}\left(t_{1}\right) & =g^{(j)}\left(t_{2}\right)-f^{(j)}\left(t_{1}\right) \\
& =g^{(j)}\left(t_{2}\right)-g^{(j)}(b)+f^{(j)}(b)-f^{(j)}\left(t_{1}\right) \\
& =\int_{b}^{t_{2}} G_{j}(\tau) d \tau+\int_{t_{1}}^{b} F_{j}(\tau) d \tau \\
& =\int_{t_{1}}^{t_{2}} H_{j}(\tau) d \tau .
\end{aligned}
$$

From this, we conclude that

$$
h^{(j)} \in A C(\overline{(a, T)}) \quad \text { for all } j \in\{0, \ldots, k-1\} .
$$

Hence, in view of (1.4), to complete the proof of the desired result it remains to check that $\Theta_{k, \alpha, h, T} \in L^{1}((a, T))$, for every $T>a$, namely that

$$
\int_{a}^{T} \frac{\left|h^{(k)}(\tau)\right|}{(T-\tau)^{\alpha-k+1}} d \tau<+\infty .
$$

We remark that here $h^{(k)}$ is intended in the Lebesgue sense, being $h^{(k-1)} \in A C(\overline{(a, T)})$, due to (A.6). Hence, in the setting of (A.5), we have that $h^{(k)}=H_{k-1}$ and therefore

$$
\int_{a}^{T} \frac{\left|h^{(k)}(\tau)\right|}{(T-\tau)^{\alpha-k+1}} d \tau=\int_{a}^{T} \frac{\left|H_{k-1}(\tau)\right|}{(T-\tau)^{\alpha-k+1}} d \tau
$$

Consequently, if $T \leq b$ we have that

$$
\int_{a}^{T} \frac{\left|h^{(k)}(\tau)\right|}{(T-\tau)^{\alpha-k+1}} d \tau=\int_{a}^{T} \frac{\left|F_{k-1}(\tau)\right|}{(T-\tau)^{\alpha-k+1}} d \tau=\left\|\Theta_{k, \alpha, f, T}\right\|_{L^{1}(a, T)},
$$

which is finite since $f \in C_{a}^{k, \alpha}$.

If instead $T>b$, we have that

$$
\begin{aligned}
\int_{a}^{T} \frac{\left|h^{(k)}(\tau)\right|}{(T-\tau)^{\alpha-k+1}} d \tau & =\int_{a}^{b} \frac{\left|F_{k-1}(\tau)\right|}{(T-\tau)^{\alpha-k+1}} d \tau+\int_{b}^{T} \frac{\left|G_{k-1}(\tau)\right|}{(T-\tau)^{\alpha-k+1}} d \tau \\
& \leq \int_{a}^{b} \frac{\left|F_{k-1}(\tau)\right|}{(b-\tau)^{\alpha-k+1}} d \tau+\int_{b}^{T} \frac{\left|G_{k-1}(\tau)\right|}{(T-\tau)^{\alpha-k+1}} d \tau \\
& =\left\|\Theta_{k, \alpha, f, b}\right\|_{L^{1}(a, b)}+\left\|\Theta_{k, \alpha, g, T}\right\|_{L^{1}(b, T)}
\end{aligned}
$$

which are finite since $f \in C_{a}^{k, \alpha}$ and $g \in C_{b}^{k, \alpha}$. This completes the proof of (A.7) and of the desired result. 
With this, we can obtain a counterpart of Lemma A.1 (which is not explicitly used here, but that can be useful for further investigations), as follows:

Lemma A.3. Let $a \in \mathbb{R} \cup\{-\infty\}$ and $b \in(a,+\infty)$. Let $I \Subset(b,+\infty)$ be an interval. Let $k \in \mathbb{N}$ and $\alpha \in(k-1, k)$, and assume that $u \in C_{b}^{k, \alpha}$.

Let also

$$
u_{\star}(t):= \begin{cases}u(t) & \text { if } t \in[b,+\infty), \\ \sum_{j=0}^{k-1} \frac{u^{(j)}(b)}{j !}(t-b)^{j} & \text { if } t \in(-\infty, b) .\end{cases}
$$

Then, $u_{\star} \in C_{a}^{k, \alpha}$ and $D_{a}^{\alpha} u_{\star}=D_{b}^{\alpha} u$ in $I$.

Proof. We apply Lemma A.2 with

$$
f(t):=\sum_{j=0}^{k-1} \frac{u^{(j)}(b)}{j !}(t-b)^{j}
$$

$g(t):=u(t)$, and $h(t):=u_{\star}(t)$. Notice that, in this setting, for each $j \in\{0, \ldots, k-1\}$, we have that $f^{(j)}(b)=u^{(j)}(b)=g^{(j)}(b)$, and therefore condition (A.3) is fulfilled. Hence, the use of Lemma A.2 gives that $u_{\star} \in C_{a}^{k, \alpha}$, as desired. In addition, we have that $u_{\star}^{(k)}=0$ in $(-\infty, b)$ and therefore, if $t \in I$,

$$
\int_{a}^{t} \frac{u_{\star}^{(k)}(\tau)}{(t-\tau)^{\alpha-k+1}} d \tau=\int_{b}^{t} \frac{u_{\star}^{(k)}(\tau)}{(t-\tau)^{\alpha-k+1}} d \tau=\int_{b}^{t} \frac{u^{(k)}(\tau)}{(t-\tau)^{\alpha-k+1}} d \tau
$$

which says that $D_{a}^{\alpha} u_{\star}(t)=D_{b}^{\alpha} u(t)$.

\section{References}

[AS64] Milton Abramowitz and Irene A. Stegun, Handbook of mathematical functions with formulas, graphs, and mathematical tables, National Bureau of Standards Applied Mathematics Series, vol. 55, For sale by the Superintendent of Documents, U.S. Government Printing Office, Washington, D.C., 1964. MR0167642

[Alm17] Ricardo Almeida, A Caputo fractional derivative of a function with respect to another function, Commun. Nonlinear Sci. Numer. Simul. 44 (2017), 460-481, DOI 10.1016/j.cnsns.2016.09.006. MR3554830

[ADPM11] Luigi Ambrosio, Giuseppe Da Prato, and Andrea Mennucci, Introduction to measure theory and integration, Appunti. Scuola Normale Superiore di Pisa (Nuova Serie) [Lecture Notes. Scuola Normale Superiore di Pisa (New Series)], vol. 10, Edizioni della Normale, Pisa, 2011. MR3379909

[Buc17] Claudia Bucur, Local density of Caputo-stationary functions in the space of smooth functions, ESAIM Control Optim. Calc. Var. 23 (2017), no. 4, 1361-1380, DOI 10.1051/cocv/2016056. MR3716924

[Cap08] Michele Caputo, Linear models of dissipation whose $Q$ is almost frequency independent. II, Fract. Calc. Appl. Anal. 11 (2008), no. 1, 4-14. Reprinted from Geophys. J. R. Astr. Soc. 13 (1967), no. 5, 529-539. MR2379269

[CDV] Alessandro Carbotti, Serena Dipierro, and Enrico Valdinoci, Local density of solutions of time and space fractional equations, In progress. 
[DSV17] Serena Dipierro, Ovidiu Savin, and Enrico Valdinoci, All functions are locally s-harmonic up to a small error, J. Eur. Math. Soc. (JEMS) 19 (2017), no. 4, 957-966, DOI 10.4171/JEMS/684. MR3626547

[DSV18] — Local approximation of arbitrary functions by solutions of nonlocal equations, J. Geom. Anal., posted on 2018, DOI 10.1007/s12220-018-0045-z.

[Sam02] Stefan G. Samko, Hypersingular integrals and their applications, Analytical Methods and Special Functions, vol. 5, Taylor \& Francis, Ltd., London, 2002. MR1918790

[Tar13] Vasily E. Tarasov, No violation of the Leibniz rule. No fractional derivative, Commun. Nonlinear Sci. Numer. Simul. 18 (2013), no. 11, 2945-2948, DOI 10.1016/j.cnsns.2013.04.001. MR3072517 